\title{
Equilibrium First-Order Melting and Second-Order Glass Transitions of the Vortex Matter in $\mathrm{Bi}_{2} \mathrm{Sr}_{2} \mathrm{CaCu}_{2} \mathrm{O}_{8}$
}

\author{
H. Beidenkopf,, , : N. Avraham,${ }^{1}$ Y. Myasoedov,${ }^{1}$ H. Shtrikman, ${ }^{1}$ E. Zeldov, ${ }^{1}$ \\ B. Rosenstein, ${ }^{1,2}$ E.H. Brandt, ${ }^{3}$ and T. Tamegai ${ }^{4}$ \\ ${ }^{1}$ Department of Condensed Matter Physics, Weizmann Institute of Science, Rehovot 76100, Israel \\ ${ }^{2}$ National Center for Theoretical Sciences and Electrophysics Department, \\ National Chiao Tung University, Hsinchu 30050, Taiwan, R.O.C. \\ ${ }^{3}$ Max-Planck-Institut für Metallforschung, Heisenbergstr. 3, D-70506 Stuttgart, Germany \\ ${ }^{4}$ Department of Applied Physics, The University of Tokyo, Hongo, Bunkyo-ku, Tokyo 113-8656, Japan
}

(Dated: June 26, 2018)

\begin{abstract}
The thermodynamic $H-T$ phase diagram of $\mathrm{Bi}_{2} \mathrm{Sr}_{2} \mathrm{CaCu}_{2} \mathrm{O}_{8}$ was mapped by measuring local equilibrium magnetization $M(H, T)$ in presence of vortex 'shaking'. Two equally sharp first-order magnetization steps are revealed in a single temperature sweep, manifesting a liquid-solid-liquid sequence. In addition, a second-order glass transition line is revealed by a sharp break in the equilibrium $M(T)$ slope. The first- and second-order lines intersect at intermediate temperatures, suggesting the existence of four phases: Bragg glass and vortex crystal at low fields, glass and liquid at higher fields.
\end{abstract}

PACS numbers: 74.25.Qt, 74.25.Dw, 74.72.Hs, 64.70.Pf

The magnetic field vs. temperature $(H-T)$ phase diagram of the vortex matter in high-temperature superconductors, and in $\mathrm{Bi}_{2} \mathrm{Sr}_{2} \mathrm{CaCu}_{2} \mathrm{O}_{8}$ (BSCCO) in particular, has drawn extensive scientific attention [1]. The commonly cited thermodynamic phase diagram of BSCCO currently consists of a single unified first-order (FO) melting line. It separates the low-field quasi long-range ordered Bragg glass (BrG) phase from the high-field liquid and glass phases $[1,2,3,4,4,5,6]$. It is not clear, however, whether the two high-field disordered phases are thermodynamically distinct, or rather reflect a gradual dynamic cross-over from liquid into a frozen, pinned state upon cooling [7, 8]. In this letter we show that the equilibrium phase diagram of the vortex matter is indeed more diverse than the one usually considered.

Experimentally, one of the main obstacles in mapping the low-temperature thermodynamics of the vortex matter is its logarithmically slow relaxation rate. Consequently, the phase diagram has been studied in the past mostly through dynamic phenomena. Two prime examples are the irreversibility line itself, marking the onset of hysteresis, and the second magnetization peak (SMP), observed along such hysteretic magnetization loops [9].

Recently, though, vortex 'shaking' was shown to be extremely effective in catalyzing relaxation at low temperatures 2, 10, 11]. Its application unveiled the inverse melting and the thermodynamic FO transition as the phenomenon underlying the non-equilibrium SMP 2. The 'shaking' method employs the segregated penetration of an in-plane field component into the highly anisotropic BSCCO samples in the form of Josephson vortices, which are confined in between the $\mathrm{CuO}_{2}$ planes. In the presence of an $a c$ in-plane field, the Josephson vortices instantaneously bisect the pancake vortex (PV) stacks on their passage, interacting mainly with adjacent
PVs, while most of the PVs in the stack remain at rest 12. These occasional interactions agitate pinned PVs, assisting them in assuming their equilibrium configuration.

Within the present study we performed local magnetization measurements by field and temperature sweeps, while utilizing the 'shaking' method, to map the equilibrium phase diagram of the vortex matter. The crossmapping of the FO melting line along both sweeping directions shows an excellent agreement. Temperature sweeps provided particularly sharp features, with which we demonstrate a liquid-solid-liquid sequence of phases. Ertaş and Nelson 13] have predicted such a liquid-solidliquid sequence to occur within a single temperature sweep, but it was never observed experimentally. We further find evidence of a novel second order (SO) phase transition within the vortex solid phase, which bears important consequences regarding the nature of the $\mathrm{BrG}$ phase.

The reported results were obtained with a slightly over-doped BSCCO crystal with $T_{\mathrm{c}} \approx 90 \mathrm{~K}$ grown by the travelling solvent floating zone method [14]. This specific sample was polished into a triangular prism of base $660 \times 270 \mu \mathrm{m}^{2}$ and height $70 \mu \mathrm{m}[15$. (other samples yielded similar results, to be presented elsewhere). The sample was attached onto an array of eleven $10 \times 10 \mu \mathrm{m}^{2}$ GaAs/AlGaAs Hall sensors. In all measurements taken below $60 \mathrm{~K}$ the sample was subject to a $10 \mathrm{~Hz}$ in-plane ac field of amplitude 350 Oe, which was aligned parallel to the planes to an accuracy of a few millidegrees. Note that according to the anisotropic scaling theory [1] this in-plane field is effectively attenuated by a factor $\gamma \simeq 200$ - the anisotropy constant in BSCCO. We found that at higher temperatures 'shaking' had no effect on the FO transition besides a small broadening (see below). 
The field sweep mapping of the FO melting line is shown in Fig. 1] The collapse of the hysteretic magnetization into a reversible behavior upon 'shaking' is demonstrated in Fig. 17 taken at $32 \mathrm{~K}$. A reversible magnetization step appears instead of the SMP. To better resolve the step we plot in Fig. 10 the derivative of the measured induction with respect to applied field $\mathrm{d} B / \mathrm{d} H$. The FO transition thus appears reversibly as a $\delta$-like peak (red) on top of the $\mathrm{d} B / \mathrm{d} H \approx 1$ (blue) background. Figure 11. shows a color scheme of the derivatives $d B / d H$ measured by field sweeps within the temperature range 28$80 \mathrm{~K}$. The individual melting peaks combine to give the locus of the FO transition line $H_{\mathrm{m}}(T)$. Its negative slope at elevated temperatures becomes positive below $38 \mathrm{~K}$. This non-monotonic behavior marks the change in the character of the transition from thermally-induced above the extremum to disorder-driven in the inverse melting region [5, 6, 13].

In addition to the field sweeps, our experimental setup also enables temperature sweeps in the presence of vortex 'shaking'. It thus allows to measure directly the temperature dependence of the equilibrium magnetization at a constant applied c-axis field. At fields slightly lower than 390 Oe (e.g. at 380 Oe along the dashed line in Fig. [2]) these sweeps should cross the melting line twice. Remarkably, the measured local induction in Fig. 20 indeed shows two very clear and opposite equilibrium magnetization steps on both descending and ascending sweeps. Note that for clarity we have subtracted from the data a

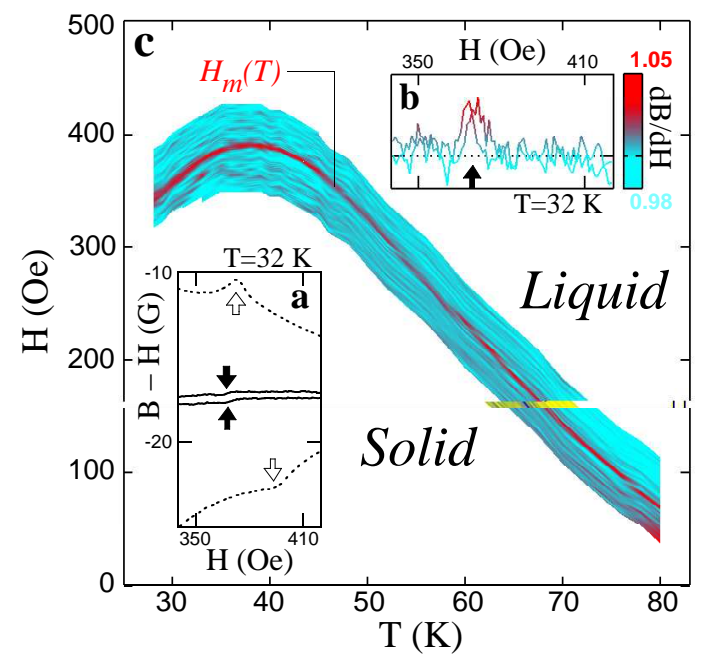

FIG. 1: (color online) (a) $32 \mathrm{~K}$ field sweeps with (solid) and without (dotted) an in-plane $350 \mathrm{Oe}-10 \mathrm{~Hz}$ 'shaking' field. Reversible steps in magnetization (solid arrows) appear instead of the hysteretic SMP (open arrows) upon shaking. (b) The derivative of the induction with respect to applied field $\mathrm{d} B / \mathrm{d} H$ at $32 \mathrm{~K}$ as a color scheme. The first-order transition appears as a paramagnetic peak (red) on top of the $\mathrm{d} B / \mathrm{d} H \approx 1$ background (blue). (c) Successive mapping of the first-order melting line $H_{\mathrm{m}}(T)$ measured by field sweeps. linear slope $\alpha T$. It is contributed both by the slight temperature dependence of the Hall coefficients of the sensors and by the linear term of the magnitude of the diamagnetic equilibrium magnetization, which monotonically decreases with temperature. This is the first observation of two FO transitions obtained in a single temperature sweep. Moreover, the two steps are equally sharp and with comparable heights of about $0.15 \mathrm{G}$. This demonstrates that the thermally- and disorder-driven processes, responsible for the melting and the inverse melting respectively, are equivalent mechanisms leading to a FO destruction of the quasi-ordered vortex solid.

The temperatures at which the magnetization steps appear along the temperature sweep of Fig. $2 \mathrm{~b}$ are in complete agreement with the melting behavior deduced from field sweeps (dotted lines). Therefore, the transition line in Fig. $2 \mathrm{a}$ is independent of the specific $H-T$ path along which it is approached - a mandatory equilibrium property. The remaining small hysteresis of 0.1 to $0.2 \mathrm{G}$ between downward and upward sweeps apparently results from surface barrier effects, while the vortices in the bulk are well equilibrated by the 'shaking'. The finite widths (about $0.7 \mathrm{~K}$ ) that the melting steps attain are mainly due to a spatial and a temporal averaging mechanisms. The first is introduced by the sensor's finite active area that averages over the propagating melting front [16], which results from the spatially inhomogeneous equilibrium magnetization profile [17]. The temporal one is a

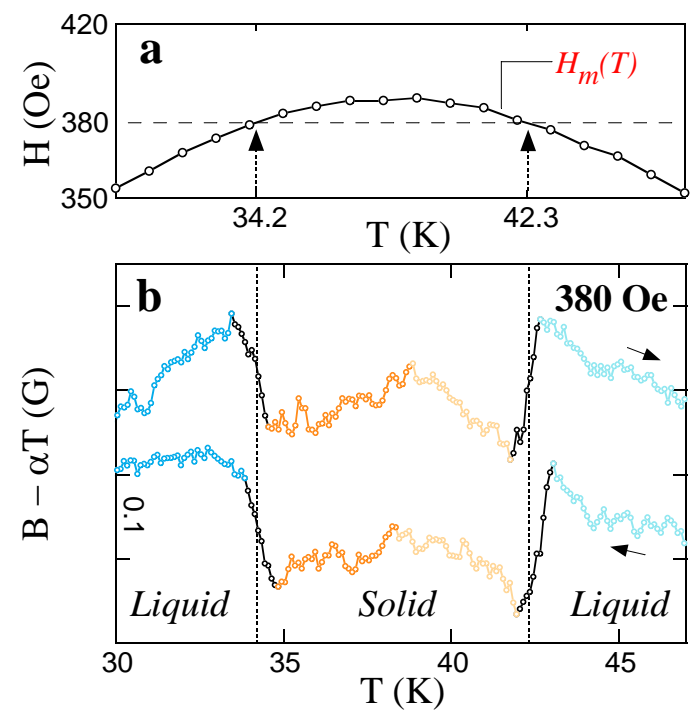

FIG. 2: (color online) (a) The FO melting line $H_{\mathrm{m}}(T)$ mapped via field sweeps (open circles). (b) Local induction $B(T)$ in the presence of 'shaking', measured upon temperature sweep at 380 Oe along the dashed line in (a). A linear slope $\alpha T$ was subtracted for clarity. The two equally narrow FO magnetization steps (black segments) show a liquid-solid-liquid sequence. The temperatures at which the phase transitions occur coincide with those, extracted from field sweeps (doted lines). The color code reflects different phases in Fig. 4 
by-product of the 'shaking' technique. The in-plane field component is known to slightly reduce the melting temperature 12, 18, 19]. Consequently, our time averaged measurement in the presence of the ac in-plane 'shaking' field results in an additional broadening due to the instantaneous periodic shift of the effective local melting temperature.

We thus turn to report the detection of a novel phase transition, whose signature is a distinct break in the slope of the magnetization $M(T)$. It is visible around $37 \mathrm{~K}$ in Fig. [2] at 380 Oe, and becomes much more pronounced at fields further away from the extremum of the FO melting line, as depicted by Fig. 3 . The 420 Oe temperature sweep (Fig. [3 $\mathrm{k}$ ) does not intersect with the FO line, hence no steps appear in the local induction. Nevertheless, a sharp break in slope is clearly resolved along both descending and ascending temperature sweeps at $T_{\mathrm{g}}$. A sharp reversible break in the induction slope appears also in the 350 Oe temperature sweep of Fig. 3p (dotted line) in between the two melting steps, hence within the solid phase. This non-analytic behavior is emphasized by the sharp step in the derivative $\mathrm{d} B / \mathrm{d} T$ shown in the insets. These kinks were found also in other samples and at various Hall-sensor locations, and did not depend on the sweeping rate. We thus conclude, that this break in slope of the equilibrium magnetization $M(T)$ indicates a thermodynamic $S O$ phase transition.

Mapping of both the first-order $H_{\mathrm{m}}(T)$ and secondorder $H_{\mathrm{g}}(T)$ transition lines onto the equilibrium $H-T$ phase diagram is given in Fig. [4 The SO line (solid dots) intersects the melting curve (open circles), and shows weak field dependence throughout the mapped region (and therefore cannot be readily observed by field sweeps). The resulting phase diagram consists of four distinct thermodynamic phases.

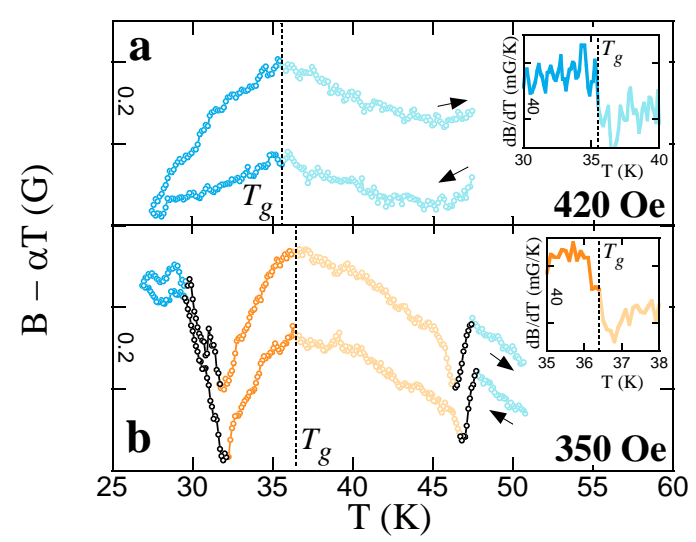

FIG. 3: (color online) Local induction $B(T)$, measured along temperature sweeps while 'shaking'. A reversible sharp break in the slope (at the dotted lines) appears both above (a $420 \mathrm{Oe}$ ) and below ( $\mathrm{b}-350 \mathrm{Oe}$ ) the melting line $H_{\mathrm{m}}(T)$. The insets show a corresponding step in the derivative $\mathrm{d} B / \mathrm{d} T$, signifying a thermodynamic second-order phase transition.
The high-field part of the novel SO line can be naturally identified with the long sought glass transition line. It asserts that the low-temperature glass phase is indeed thermodynamically distinct from the high-temperature liquid one. Several experimental studies have observed bulk irreversibility features in BSCCO, which appeared above $H_{\mathrm{m}}(T)$ at about $35 \mathrm{~K}[20,21]$. However, all these studies probed dynamic or non-equilibrium vortex properties. The glass line of Fig. 4 is the first experimental evidence of such a thermodynamic transition in BSCCO.

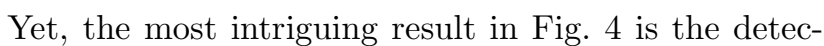
tion of the SO line within the vortex solid region (orange). This implies that two distinct thermodynamic phases are present in the low-field region below $H_{\mathrm{m}}(T)$, contrary to the common belief that a single $\mathrm{BrG}$ phase prevails throughout this part of the phase diagram. A number of previous studies indicated a depinning line of similar topology within the $\mathrm{BrG}$ below $H_{\mathrm{m}}(T)$ 20]. However, all these measurements probed only the non-equilibrium properties, which were consistent with the existing theoretical dynamic predictions and simulations of depinning 13, 22]. In contrast, the present finding of a thermodynamic line requires a more fundamental reconsideration.

It is interesting to note that in $\mathrm{YBa}_{2} \mathrm{Cu}_{3} \mathrm{O}_{7}$ crystals a thermodynamic signature of a $\mathrm{SO}$ transition within the liquid phase has been reported [23]. There, however, the SO line emanates from the upper critical point of the FO line, directly extending it to higher fields. This topology is consistent with several dynamic measurements in $\mathrm{YBa}_{2} \mathrm{Cu}_{3} \mathrm{O}_{7}$ 24, 25], although alternative topologies have been also suggested [26, 27]. In contrast, our thermodynamic data of BSCCO show that the SO

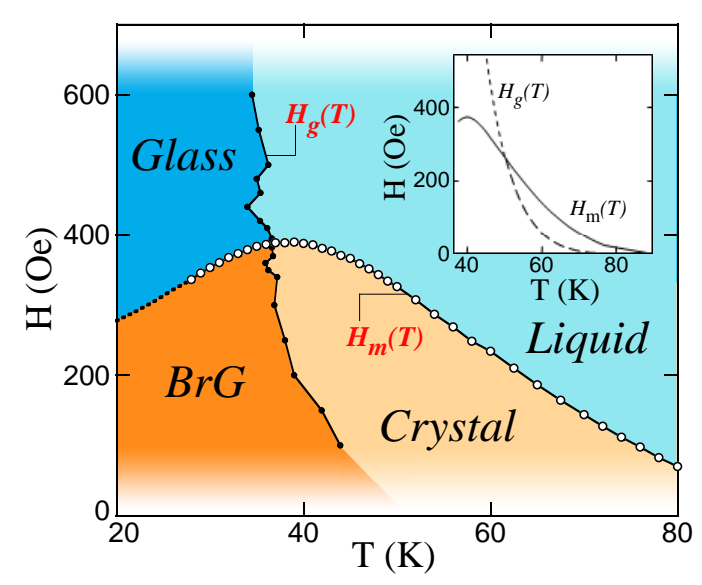

FIG. 4: (color online) The thermodynamic phase diagram of BSCCO accommodates four distinct phases, separated by a first-order melting line $H_{\mathrm{m}}(T)$ (open circles), which is intersected by the second-order glass line $H_{\mathrm{g}}(T)$ (solid dots). The inset plots an equivalent phase diagram, calculated based on Ref. 31, consisting of a second-order replica symmetry breaking lines $H_{\mathrm{g}}(T)$ both above (dotted) and below (dashed) the first-order transition $H_{\mathrm{m}}(T)$ (solid). 
and the FO transitions are two independent lines that intersect each other nearly at a right angle.

Several theoretical studies have shown that under the elastic medium approximation quasi long-range order of the vortex lattice is still retained in the presence of quenched disorder, giving rise to the BrG phase [28, 29]. This phase was found to be stable at all temperatures (as long as topological excitations are excluded) in systems of dimensionality greater than two and lower than four. This is probably the reason why a non-topological thermodynamic phase transition of the BrG phase was hardly ever considered in 3D models. An exception is a Josephson-glass line that was suggested to exist within the $\mathrm{BrG}$ region 30]. Still, the general belief is that the BrG phase is robust until dislocations proliferate, which gives rise to the $\mathrm{FO}$ phase transition $[6,8,28$. In $2 \mathrm{D}$ systems, however, the BrG models did find a possible finitetemperature depinning transition, above which disorder is no longer relevant. Therefore, the observed SO transition could be accounted for within these models only in the extreme case of vanishing coupling between the superconducting layers.

In contrast, in a recent theoretical work 31] the free energy of the vortex matter in the presence of quenched disorder was explicitly calculated under the lowest-Landaulevel approximation in a 3D model. It was found that two transitions are present: a FO melting, at which the quasi long-range order is destroyed, and a SO glass transition, below which the replica symmetry is spontaneously broken. The inset of Fig. 4 shows the phase diagram, calculated using a similar effective 2D model with parameters optimized for BSCCO [32]. The calculations reproduce the measured features very well. Amongst them are the melting and inverse-melting behavior, the discontinuity of the magnetization slope $\mathrm{d} M / \mathrm{d} T$ at the glass transition, and the $H_{\mathrm{g}}(T)$ line itself, which resides at slightly higher temperatures as compared to experiment. In addition, the calculations show that the portions of the $H_{\mathrm{g}}(T)$ line, lying above and below the FO transition $H_{\mathrm{m}}(T)$ (dotted and dashed lines, respectively), are slightly shifted from each other. Therefore, they do not cross the melting line at a single point, but rather form two closely located tricritical points along it. This minute shift can hardly be seen in the inset of Fig. 4 and is below our current experimental resolution.

Within this model the high-field glass phase and the low-field BrG are strongly pinned and replica symmetry broken, whereas the two high-temperature phases are replica symmetric and thus reversible. This conclusion of reversibility is consistent also with the existing dynamic measurements [20]. It is therefore tempting to speculate that the phase above $H_{\mathrm{g}}(T)$ and below $H_{\mathrm{m}}(T)$ should acquire a true crystalline order. However, since Rosenstein and Li did not calculate the structure factor and our measurements do not probe this quantity, the proposed vortex crystal phase certainly calls for further experimen- tal and theoretical investigations.

In summary, we present thermodynamic evidence of a possibly second-order glass transition line that splits the quasi-ordered vortex solid into two distinct phases. By comparing the results with existing dynamic measurements and a new theoretical study, we suggest that the two phases are BrG and a vortex crystal. The glass line crosses the first-order melting line near its extremum and extends to higher fields, giving rise to two thermodynamically distinct disordered phases - a glass and a liquid.

We thank D. Li, V.M. Vinokur, and B. Horovitz for stimulating discussions. This work was supported by the Israel Science Foundation Center of Excellence, by the German-Israeli Foundation G.I.F., by the Minerva Foundation, Germany, and by Grant-in-aid for Scientific Research from the Ministry of Education, Culture, Sports, Science, and Technology, Japan. BR acknowledges the support of the Albert Einstein Minerva Center for Theoretical Physics and EZ the US-Israel Binational Science Foundation (BSF).

* Electronic address: haim.beidenkopf@weizmann.ac.il

[1] G. Blatter and V.B. Geshkenbein, The Physics of Superconductors (Springer, 2003), vol. I, chap. 10, p. 725.

[2] N. Avraham et al., Nature 411, 451 (2001).

[3] Y. Radzyner, A. Shaulov, and Y. Yeshurun, Phys. Rev. B 65, 100513(R) (2002).

[4] V. Vinokur et al., Physica C 295, 209 (1998).

[5] G.P. Mikitik and E.H. Brandt, Phys. Rev. B 68, 054509 (2003); J. Kierfeld and V. Vinokur, Phys. Rev. B 69, 24501 (2004).

[6] T. Giamarchi and S. Bhattacharya, High Magnetic Fields: Applications in Condensed Matter Physics, Spectroscopy (Springer, 2002), p. 314.

[7] C. Reichhardt, A. van Otterlo, and G.T. Zimányi, Phys. Rev. Lett. 84, 1994 (2000).

[8] Y. Nonomura and X. Hu, Phys. Rev. Lett. 86, 5140 (2001); P. Olsson and S. Teitel, Phys. Rev. Lett. 87, 137001 (2001).

[9] B. Khaykovich et al., Phys. Rev. Lett. 76, 2555 (1996).

[10] M. Willemin et al., Phys. Rev. Lett. 81, 4236 (1998).

[11] G.P. Mikitik and E.H. Brandt, Phys. Rev. B 69, 134521 (2004).

[12] A.E. Koshelev, Phys. Rev. Lett. 83, 187 (1999).

[13] D. Ertaş and D.R. Nelson, Physica C 272, 79 (1996).

[14] N. Motohira et al., J. Ceram. Soc. Jpn. Int. 97, 994 (1989).

[15] D. Majer, E. Zeldov, and M. Konczykowski, Phys. Rev. Lett. 75, 1166 (1995).

[16] A. Soibel et al., Nature 406, 282 (2000).

[17] E. Zeldov et al., Phys. Rev. Lett. 73, 1428 (1994).

[18] S. Ooi et al., Phys. Rev. Lett. 82, 4308 (1999).

[19] B. Schmidt et al., Phys. Rev. B 55, R8705 (1997).

[20] D.T. Fuchs et al., Phys. Rev. Lett. 80, 4971 (1998); C.D. Dewhurst and R.A. Doyle, Phys. Rev. B 56, 10832 (1997); Y. Yamaguchi et al., Phys. Rev. B 63, 014504 (2000); S. Ooi, T. Mochiku, and K. Hirata, Physica C 378, 523 (2002); Y. Matsuda et al., Phys. Rev. Lett. 
78, 1972 (1997); S. Ooi, T. Shibaushi, and T. Tamegai, Physica B 284, 775 (2000).

[21] T. Shibauchi et al., Phys. Rev. Lett. 83, 1010 (1999).

[22] R. Sugano et al., Physica C 357, 428 (2001).

[23] F. Bouquet et al., Nature 411, 448 (2001).

[24] H. Safar et al., Phys. Rev. Lett. 70, 3800 (1993).

[25] W.K. Kwok et al., Phys. Rev. Lett. 84, 3706 (2000).

[26] K. Shibata et al., Phys. Rev. B 66, 214518 (2002).

[27] B.J. Taylor et al., Phys. Rev. B 68, 054523 (2003).
[28] T. Giamarchi and P. LeDoussal, Phys. Rev. B 52, 1242 (1995).

[29] T. Nattermann, Phys. Rev. Lett. 64, 2454 (1990).

[30] B. Horovitz and T.R. Goldin, Phys. Rev. Lett. 80, 1734 (1998); B. Horovitz, Phys. Rev. B 72, 024519 (2005).

[31] D. Li and B. Rosenstein, Phys. Rev. Lett. 90, 167004 (2003); D. Li and B. Rosenstein, cond-mat/0411096.

[32] B. Rosenstein and D. Li, unpublished. 